\title{
Formation control with mismatched compasses
}

\author{
Ziyang Meng ${ }^{\mathrm{a}}$, Brian D.O. Anderson ${ }^{\mathrm{b}}$, Sandra Hirche ${ }^{\mathrm{c}}$ \\ a State Key Laboratory of Precision Measurement Technology and Instruments, Department of Precision Instrument, Tsinghua University, \\ Beijing 100084, China \\ ${ }^{\mathrm{b}}$ The Australian National University and National ICT Australia, Canberra ACT 2600, Australia \\ ${ }^{\mathrm{c}}$ Institute for Information-Oriented Control, Technische Universität München, D-80290 Munich, Germany
}

\section{A R T I C L E I N F O}

\section{Article history:}

Received 8 December 2014

Received in revised form

6 October 2015

Accepted 13 February 2016

\section{Keywords:}

Formation control

Mismatched compasses

Estimation and compensation algorithms

\begin{abstract}
A B S T R A C T
This article addresses the formation control problem with mismatched compasses. Depending on the sensing and communication technology, compass mismatches may arise due to biases in measurement, drift in inertial sensing despite initial alignment, and even spatial variations in the earth's magnetic field. To illustrate the key concepts underlying what happens, we first consider the two agent case and show that the agents converge to a fixed, but distorted formation exponentially fast. In contrast to the matched compass case, the formation is not asymptotically stationary. The distance error and the angular error between the actual final formation and the desired formation are explicitly given, as is the steady state velocity of the formation. The case of time-varying mismatched compasses is also studied. Based on the results, we then propose estimators to obtain the mismatched angle, which allow a compensation algorithm to be proposed such that the desired formation shape is achieved. Finally, the extensions to the $n$ agent case are also considered and similar phenomena are encountered. Simulations are provided to validate the theoretical results.
\end{abstract}

(c) 2016 Elsevier Ltd. All rights reserved.

\section{Introduction}

The formation control problem for multi-agent systems has received increasing attention during the last decade due to its broad applications in spacecraft formation flying, search and rescue, and formation control of mobile robots (Cortes, Martinez, \& Bullo, 2006; He, Qian, Lam, Chen, Han \& Kurths, 2015; Meng, Anderson, \& Hirche, 2015; Sieber, Deroo, \& Hirche, 2013; Yang, Roy, Wan, \& Saberi, 2011; Zavlanos \& Pappas, 2008). There are many variations on the formation control problem, including problems with a leader or without a leader (Ren, 2007; Shi \& Hong, 2009), problems with underlying graph structure which

\footnotetext{
NICTA is supported by the Australian Government under the ICT Centre of Excellence Program. The work was also supported by National Natural Science Foundation of China under Grants 61503249 and 61403392, the Australian Research Council under grants DP110100538 and DP130103610, and the Alexander von Humboldt Foundation of Germany. The material in this paper was partially presented at the 54th Conference on Decision and Control, December 15-18, 2015, Osaka, Japan (Meng, Anderson, and Hirche 2015). This paper was recommended for publication in revised form by Associate Editor Tamas Keviczky under the direction of Editor Christos G. Cassandras.

E-mail addresses: ziyangmeng@mail.tsinghua.edu.cn (Z. Meng), brian.anderson@anu.edu.au (B.D.O. Anderson), hirche@tum.de (S. Hirche).
}

is directed or undirected (Hatano \& Mesbahi, 2005; Moreau, 2005), problems in which the formation is achieved with velocity consensus leading to a moving final formation or solutions where the final formation is stationary (Jiang, Deghat, \& Anderson, 2013; Lin, Broucke, \& Francis, 2004). One particular distinction is between securing a formation with both a prescribed shape and a prescribed orientation, as opposed to simply aiming for a prescribed shape. Seeking a prescribed shape with prescribed orientation is in fact one of the easier problems. It can be solved using a linear consensus-based algorithm, where the control input is a combination of the neighbor-based relative position term and a nonzero bias term representing the formation objective (Fax \& Murray, 2004; Olfati-Saber, Fax, \& Murray, 2007). This is contrasted with an approach for shape control without orientation which uses gradient-based control, grounded in the theory of graph rigidity and often derived from system structural potentials (Cao, Morse, Yu, Anderson, \& Dasgupta, 2011; Krick, Broucke, \& Francis, 2009). In the gradient-based approach, agents do need again to measure relative positions, but only in a local coordinate basis associated with the measuring agent, which does not have to be directionally aligned with the coordinate bases of other agents. In contrast, the consensus-based approach requires all agents to have knowledge of where the common/global north is. Equivalently, coordinate bases of the different agents have to be directionally aligned. In 
this article, the consensus-based approach is considered. For this approach, common knowledge of where north is may sometimes be expressed by saying that all agents require a compass. In practice, they may acquire knowledge of north from inertial navigation properly initialized, or landmark data, the use of which likely requires inter-agent transmissions.

It is evident that it will often be physically unrealistic to claim that all agents have common error-free knowledge of where north is: biases can exist in instruments; drift can occur in inertial navigation systems; spatial variation can occur in the earth's magnetic field. This article explores the consequence of postulating the existence of errors in the direction of north, i.e. agents have differing views of where north is. To exhibit the key ideas, which apply to formations of any size, it is convenient to consider first a very simple case. Hence we start from the matched compass formation control for two agents in a two-dimensional plane,

$\dot{\mathbf{A}}_{1}=\left(\mathbf{A}_{2}-\mathbf{A}_{1}\right)-\mathbf{D}$,

$\dot{\mathbf{A}}_{2}=\left(\mathbf{A}_{1}-\mathbf{A}_{2}\right)+\mathbf{D}$,

where $\mathbf{A}_{1}=\left[x_{1}, y_{1}\right]^{\mathrm{T}} \in \mathbb{R}^{2}, \mathbf{A}_{2}=\left[x_{2}, y_{2}\right]^{\mathrm{T}} \in \mathbb{R}^{2}$ are the positions of agents 1 and 2 , and $\mathbf{D}=\left[d_{x}, d_{y}\right]^{\mathrm{T}} \in \mathbb{R}^{2}$ is a given desired relative position and known for each agent. The objective is to drive agents 1 and 2 to form a stationary formation in the plane such that $\mathbf{A}_{2}=\mathbf{A}_{1}+\mathbf{D}$. It is straightforward to show that $\frac{\mathrm{d}}{\mathrm{d} t}\left(\mathbf{A}_{2}-\mathbf{A}_{1}-\mathbf{D}\right)=$ $-2\left(\mathbf{A}_{2}-\mathbf{A}_{1}-\mathbf{D}\right)$. This implies that $\lim _{t \rightarrow \infty}\left(\mathbf{A}_{2}(t)-\mathbf{A}_{1}(t)\right)=$ D, $\lim _{t \rightarrow \infty} \dot{\mathbf{A}}_{1}(t)=0$, and $\lim _{t \rightarrow \infty} \dot{\mathbf{A}}_{2}(t)=0$ exponentially fast. Therefore, agents converge to the desired formation and the velocities converge to zero exponentially fast.

In considering the above simple algorithm one should notice that the algorithm is constructed based on the assumption that the relative position measurement $\mathbf{A}_{2}-\mathbf{A}_{1}$ for agent 1 and the relative position measurement $\mathbf{A}_{1}-\mathbf{A}_{2}$ for agent 2 are identical (up to the sign). However, in real systems this assumption is unlikely to be satisfied for reasons as noted above. As already indicated, it is the directional error, i.e., a compass mismatch, that will concern us. For convenience but without any loss of generality, suppose that the global coordinates coincide with the coordinate basis of agent 1. We next seek to express the equation of motion of agent 2 in global coordinates. Suppose $A_{i}$ denotes the position of agent $i, i=$ 1,2 in global coordinates and ${ }^{2} A_{i}$ denotes its position in agent 2 's coordinates. Suppose agent 2's view of north is that it is $\phi$ radians in a clockwise direction from agent 1's view, where $\phi \in(-\pi, \pi]$. An illustration is given in Fig. 1. We then know that a vector defining a line segment in global coordinates, denoted by ${ }^{1} \mathbf{W}=[x, y]^{\mathrm{T}}$ is described in agent 2's coordinate basis as ${ }^{2} \mathbf{W}=R(-\phi)^{1} \mathbf{W}$, where $R^{-1}(\phi)=R(-\phi)=\left[\begin{array}{cc}\cos \phi & \sin \phi \\ -\sin \phi & \cos \phi\end{array}\right]$ is the rotation matrix.

Then, in each agent's own coordinate basis, the actual kinematics of each agent with mismatched compasses are given by

${ }^{1} \dot{\mathbf{A}}_{1}=\mathbf{A}_{2}-\mathbf{A}_{1}-\mathbf{D}$,

${ }^{2} \dot{\mathbf{A}}_{2}=R(-\phi)\left(\mathbf{A}_{1}-\mathbf{A}_{2}\right)+\mathbf{D}$,

where $\mathbf{A}_{1}-\mathbf{A}_{2}$ is expressed in global coordinates, ${ }^{1} \dot{\mathbf{A}}_{1}$ and ${ }^{2} \dot{\mathbf{A}}_{2}$ are the velocity vectors of agents 1 and 2 expressed in each agent's own coordinate basis.

A relevant work for this problem is (Oh \& Ahn, 2014), where the authors considered that there exists the orientation mismatch of local reference frames of the agents for the formation shape control problem. A combination algorithm aimed at coordinate frame orientation alignment and formation control was proposed and the assumption was imposed that the orientation of each agent's coordinate basis can be exchanged between neighbors. Distance errors have been considered in the context of formation shape control without orientation in Belabbas, Mou, Morse, and

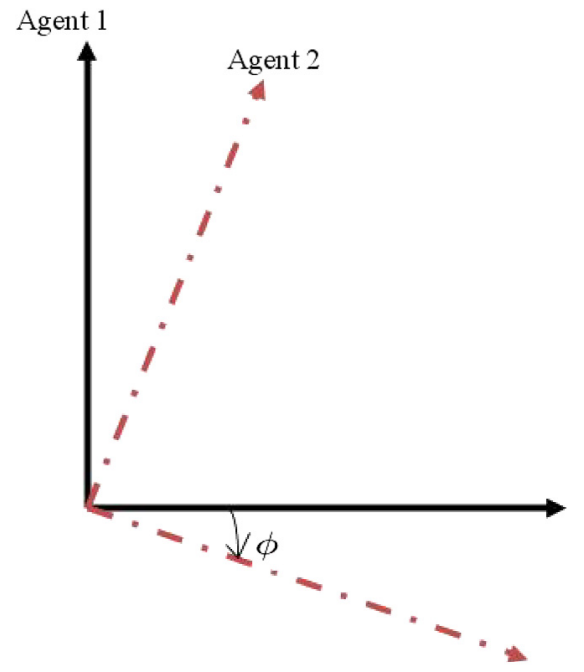

Fig. 1. Coordinates of agents 1 and 2 .

Anderson (2012) and Sun, Mou, Anderson, and Morse (2013). It was shown in Belabbas et al. (2012) that if the agents have different understandings of either the desired distance for each pair of agents or of the actual distance between them (perhaps due to measurement bias), the resulting formation shape in the limit is fixed but distorted relative to the desired shape, and generically the actual motions converge to circular closed orbits in the twodimensional plane. A nongeneric situation can also arise in which the radius of the circular orbit goes to infinity, and then the formation simply translates at a constant velocity. This actually always happens for a two-agent formation. The extension to the case of the $3 D$ tetrahedron formation shape control problem (and indeed more general 3D shapes) was considered in Sun et al. (2013) and it was shown that the motion behavior is a typical helix. It is in fact not hard to vary the conclusions of those papers and establish that a distance error for the two agent formation above leads the formation to take up a steady state spacing close to the desired distance and to move with a velocity parallel to the relative position vector at a speed proportional to the distance mismatch. One could in fact postulate directional and distance errors simultaneously. It would appear that the overall effect is just the superposition of the two individual effects.

In this article, we first focus on the compass mismatch problem for the two agent case (2) and then study the $n$ agent case. In particular, we show that the agents converge to a fixed, but (relative to the desired formation) distorted formation exponentially fast for all the cases. The shape error between the actual final formation and the desired formation is explicitly given. The case of time-varying mismatched compasses and the estimation algorithms for the mismatched angle are also studied. Based on the design of the estimators, the compensation algorithm is proposed such that the desired formation shape is achieved. We finally include discussions on the $n$ agent case where $n \geq 3$.

The organization of this article is as follows. In Section 2, we study the two agent case. Both the cases of constant error and time-varying error are considered. The estimation algorithms and compensation algorithms are proposed in Section 3. We also extend the results to the $n$ agent case where $n \geq 3$ in Sections 4 and 5. Concluding remarks are given in Section 6 .

\section{Two agent case}

Let us go back to (2) and assume that $\phi$ is constant. By noting the fact that ${ }^{1} \dot{\mathbf{A}}_{2}=R(\phi)^{2} \dot{\mathbf{A}}_{2}$, it is not hard to show that (2) can be written as

$\dot{\mathbf{A}}_{1}=\mathbf{A}_{2}-\mathbf{A}_{1}-\mathbf{D}$, 
$\dot{\mathbf{A}}_{2}=\mathbf{A}_{1}-\mathbf{A}_{2}+R(\phi) \mathbf{D}$,

where $\mathbf{A}_{1}, \mathbf{A}_{2}, \dot{\mathbf{A}}_{1}$, and $\dot{\mathbf{A}}_{2}$ are all expressed in global coordinates. We next focus on (3) and study both the intra-formation motion and whole-formation motion of the closed-loop system.

Notation. $\|x\|$ denotes the 2 -norm of a vector $x \in \mathbb{R}^{d}$. Let $f$ and $g$ be two functions defined on some subset of the real numbers. One writes $f(x)=O(g(x))$ as $x \rightarrow 0$ if and only if there exist positive real numbers $M$ and $\delta$ such that $|f(x)| \leq M|g(x)|$ for $|x|<\delta$.

\subsection{Main results}

We first establish the following result on how the formation of two agents evolves when there exist compass mismatches.

Theorem 1. Consider the mismatched formation control algorithm (2). Suppose $\phi \neq 0$ and is constant over time. It follows that [Intra-formation motion]

(I) The agents converge to a fixed formation exponentially fast. In particular, $\lim _{t \rightarrow \infty}\left(\mathbf{A}_{2}(t)-\mathbf{A}_{1}(t)\right)=\cos \frac{\phi}{2} R(\phi / 2) \mathbf{D}$.

(II) The relative velocities of the agents converge to zero exponentially fast, i.e., $\lim _{t \rightarrow \infty}\left(\mathbf{V}_{2}(t)-\mathbf{V}_{1}(t)\right)=0$, where $\mathbf{V}_{1}=$ $\left[v_{x, 1}, v_{y, 1}\right] \in \mathbb{R}^{2}$ and $\mathbf{V}_{2}=\left[v_{x, 2}, v_{y, 2}\right] \in \mathbb{R}^{2}$ are the velocities of agents 1 and 2 .

(III) If $\mathbf{D} \neq 0$, the final formation is distorted from the desired one. The distance error and the angular error between the actual final relative position and the desired one are $O\left(\phi^{2}\right)$, as $\phi \rightarrow 0$ and $\frac{|\phi|}{2}$, respectively. If $\mathbf{D}=0$, then rendezvous is still achieved, i.e., $\lim _{t \rightarrow \infty}\left(\mathbf{A}_{2}(t)-\mathbf{A}_{1}(t)\right)=0$.

[Whole-formation motion]

(IV) If $\mathbf{D} \neq 0$, the absolute velocities of each agent converge to the same nonzero constant exponentially fast. In particular, $\lim _{t \rightarrow \infty} \mathbf{V}_{1}(t)=\lim _{t \rightarrow \infty} \mathbf{V}_{2}(t)=-\sin \frac{\phi}{2}\left[\begin{array}{cc}\sin \frac{\phi}{2} & \cos \frac{\phi}{2} \\ -\cos \frac{\phi}{2} & \sin \frac{\phi}{2}\end{array}\right] \mathbf{D}$ and $\lim _{t \rightarrow \infty}\left\|\mathbf{V}_{1}(t)\right\|=\lim _{t \rightarrow \infty}\left\|\mathbf{V}_{2}(t)\right\|=O(|\phi|)$, as $\phi \rightarrow 0$. If $\mathbf{D}=0$, the absolute velocities of each agent converge to zero exponentially fast.

(V) If $\mathbf{D} \neq 0$, the agent positions $\mathbf{A}_{1}(t)$ and $\mathbf{A}_{2}(t)$ are neither convergent nor bounded. If $\mathbf{D}=0, \lim _{t \rightarrow \infty} \mathbf{A}_{1}(t)=$ $\lim _{t \rightarrow \infty} \mathbf{A}_{2}(t)=\frac{\mathbf{A}_{1}(0)+\mathbf{A}_{2}(0)}{2}$, where $\mathbf{A}_{1}(0)$ and $\mathbf{A}_{2}(0)$ are the initial states of agents 1 and 2 .

Proof. (I) Define $\mathbf{Q}=\mathbf{A}_{1}-\mathbf{A}_{2}+\frac{1}{2}(\mathbf{I}+R(\phi)) \mathbf{D}$. It follows from (3) that

$\dot{\mathbf{Q}}=-2 \mathbf{Q}$.

It then follows that $\lim _{t \rightarrow \infty}\left(\mathbf{A}_{2}(t)-\mathbf{A}_{1}(t)\right)=\overline{\mathbf{D}}$ exponentially, where $\overline{\mathbf{D}}=\frac{1}{2}(\mathbf{I}+R(\phi)) \mathbf{D}$. Straightforward calculation shows that $\overline{\mathbf{D}}=\left[\begin{array}{cc}\frac{1+\cos \phi}{2} & -\frac{\sin \phi}{2} \\ \frac{\sin \phi}{2} & \frac{1+\cos \phi}{2}\end{array}\right] \mathbf{D}=\cos \frac{\phi}{2} R(\phi / 2) \mathbf{D}$. This verifies (I).

(II) Since $\lim _{t \rightarrow \infty}\left(\mathbf{A}_{2}(t)-\mathbf{A}_{1}(t)-\overline{\mathbf{D}}\right)=0$ exponentially fast, it follows from (4) that $\lim _{t \rightarrow \infty}\left(\dot{\mathbf{A}}_{2}(t)-\dot{\mathbf{A}}_{1}(t)\right)=0$ exponentially fast. Therefore, (II) is proven.

(III) Note that the desired formation is determined by $\mathbf{D}$, but the actual final formation is determined by $\overline{\mathbf{D}} \neq \mathbf{D}$. Therefore, for the case of $\mathbf{D} \neq 0$, the final formation is distorted from the desired one. We define the distance error between the actual final relative position and the desired one as $\delta \mathbf{D}=|\|\overline{\mathbf{D}}\|-\|\mathbf{D}\||$. It is not hard to show that $\|\overline{\mathbf{D}}\|=\cos ^{2} \frac{\phi}{2}\left\|\left[\begin{array}{cc}1 & -\tan \frac{\phi}{2} \\ \tan \frac{\phi}{2} & 1\end{array}\right] \mathbf{D}\right\|=$ $\cos ^{2} \frac{\phi}{2} \sec \frac{\phi}{2}\|\mathbf{D}\|=\cos \frac{\phi}{2}\|\mathbf{D}\|$. Therefore, for small $|\phi|, \delta \mathbf{D}=(1-$ $\left.\cos \frac{\phi}{2}\right)\|\mathbf{D}\|=2 \sin ^{2}(\phi / 4)\|\mathbf{D}\| \approx \frac{\phi^{2}}{8}\|\mathbf{D}\|$. This shows that $\delta \mathbf{D}=$ $O\left(\phi^{2}\right)$, as $\phi \rightarrow 0$. In addition, by noting that $\overline{\mathbf{D}}=\cos \frac{\phi}{2} R(\phi / 2) \mathbf{D}$, we know that the angular error between the actual final relative position and the desired one is $\frac{|\phi|}{2}$. On the other hand, if $\mathbf{D}=0$, it follows from the definition of $\overline{\mathbf{D}}$ that rendezvous is still achieved, i.e., $\lim _{t \rightarrow \infty}\left(\mathbf{A}_{2}(t)-\mathbf{A}_{1}(t)\right)=0$.

(IV) It follows from (2) that $\lim _{t \rightarrow \infty} \dot{\mathbf{A}}_{1}(t)=\lim _{t \rightarrow \infty} \dot{\mathbf{A}}_{2}(t)$ $=\frac{1}{2}(R(\phi)-\mathbf{I}) \mathbf{D}=\left[\begin{array}{cc}\frac{\cos \phi-1}{2} & -\frac{\sin \phi}{2} \\ \frac{\sin \phi}{2} & \frac{\cos \phi-1}{2}\end{array}\right] \mathbf{D}=-\sin \frac{\phi}{2} \times$ $\left[\begin{array}{cc}\sin \frac{\phi}{2} & \cos \frac{\phi}{2} \\ -\cos \frac{\phi}{2} & \sin \frac{\phi}{2}\end{array}\right]$

D. This further implies when $\mathbf{D} \neq 0$ that $\lim _{t \rightarrow \infty}\left\|\dot{\mathbf{A}}_{1}(t)\right\|=\lim _{t \rightarrow \infty}\left\|\dot{\mathbf{A}}_{2}(t)\right\|=O(|\phi|)$, as $\phi \rightarrow 0$. Therefore, for the case of $\mathbf{D} \neq 0$, the absolute velocities of each agent converge to the same nonzero constant exponentially fast. For the case of $\mathbf{D}=0$, the absolute velocities of each agent converge to zero exponentially fast.

(V) For the case of $\mathbf{D} \neq 0$, the conclusions are obvious due to (IV). If $\mathbf{D}=0$, the problem reduces to a standard average consensus problem (Olfati-Saber et al., 2007).

Remark 1. When $\mathbf{D}=0$, the considered problem is reduced to a standard consensus problem. Our results show that the existence of compass mismatch does not change the qualitative behavior of reaching a consensus.

We next describe simulations which illustrate the validity of Theorem 1. We consider the case of $\mathbf{D} \neq 0$. In particular, $\mathbf{D}=[0,-5]^{\mathrm{T}}, \phi=0.2 \pi$, and the two agents start from the desired formation. Figs. 2 and 3 show the positions and velocities of the agents during the time interval $[0,10]$. The relative velocities converge to zero, which shows that the agents converge to a fixed formation. However, due to the existence of mismatched compasses, the agents keep moving with non-zero constant absolute velocities and the final formation is distorted from the desired one. This is in contrast to the case with matched compasses where the agents converge to the desired stationary formation.

\subsection{Extension to time-varying mismatched compasses}

Theorem 1 treats the case that $\phi$ is a constant. However, it is quite possible that the directional error $\phi$ is varying with respect to $t$. Therefore, in this subsection of the article, we consider the case of time-varying bounded $\phi(t)$, where the formation control algorithm becomes:

$\begin{aligned}{ }^{1} \dot{\mathbf{A}}_{1} & =\mathbf{A}_{2}-\mathbf{A}_{1}-\mathbf{D} \\ { }^{2} \dot{\mathbf{A}}_{2} & =R(-\phi(t))\left(\mathbf{A}_{1}-\mathbf{A}_{2}\right)+\mathbf{D} .\end{aligned}$

Definition 1. Consider (5). The error between the actual final formation and the desired one is called globally ultimately bounded with ultimate bound $\beta$ if there exists a positive constant $\beta$, independent of initial time $t_{0} \geq 0$, and there is $T=T(\beta) \geq 0$, independent of initial time $t_{0} \geq 0$, such that $\left\|\mathbf{A}_{2}(t)-\mathbf{A}_{1}(t)-\mathbf{D}\right\| \leq$ $\beta, \forall t \geq t_{0}+T, \forall \mathbf{A}_{1}(0), \mathbf{A}_{2}(0) \in \mathbb{R}^{2}$.

We can analogously define that the relative velocity of the agents $\mathbf{V}_{2}(t)-\mathbf{V}_{1}(t)$ is globally ultimately bounded with ultimate bound $\beta$. Next, the following result on the case of time-varying mismatched compasses is established. 


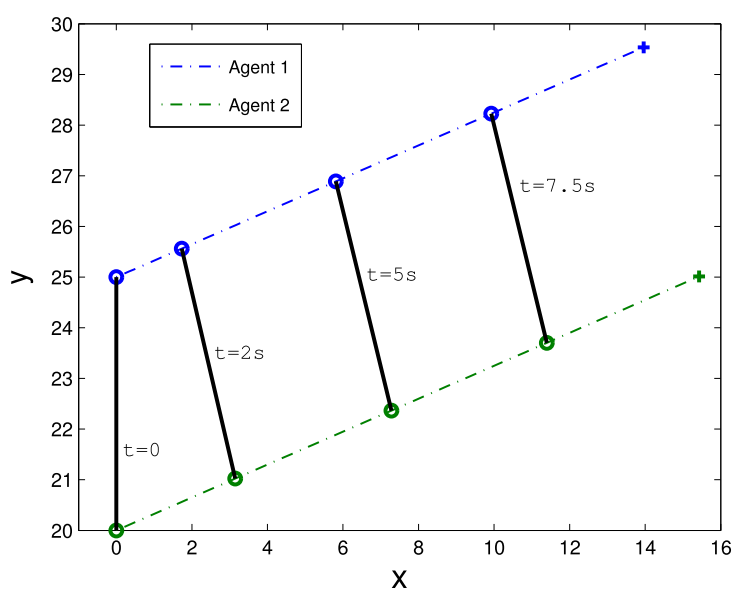

(a) The dotted lines denote the trajectories of the positions of the agents. The circles and the solid black lines denote respectively, the positions of the agents and the formation shape at $t=0 \mathrm{~s}, t=2 \mathrm{~s}$, $t=5 \mathrm{~s}$, and $t=7.5 \mathrm{~s}$.

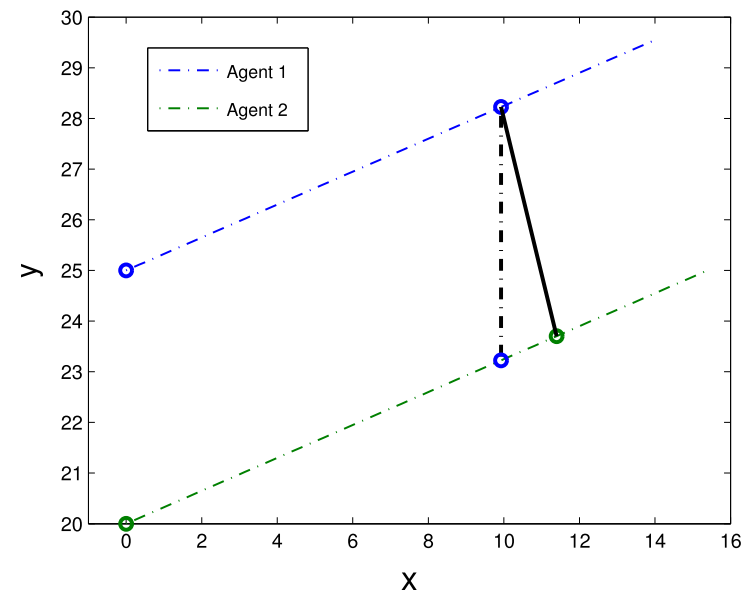

(b) The solid black line and the dotted black line denote respectively, the actual formation shape and desired formation shape at $t=7.5 \mathrm{~s}$.

Fig. 2. Two agent case with $\mathbf{D} \neq 0$.
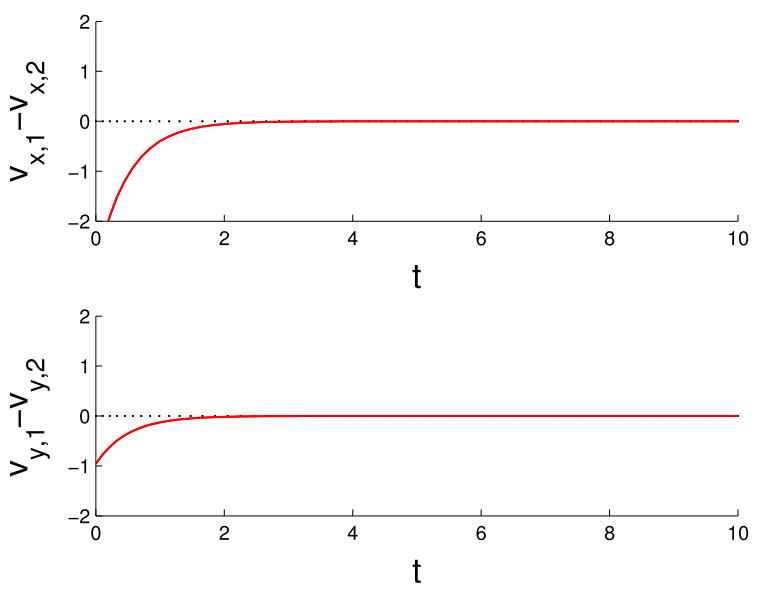

(a) The convergence of relative velocities of the agents.
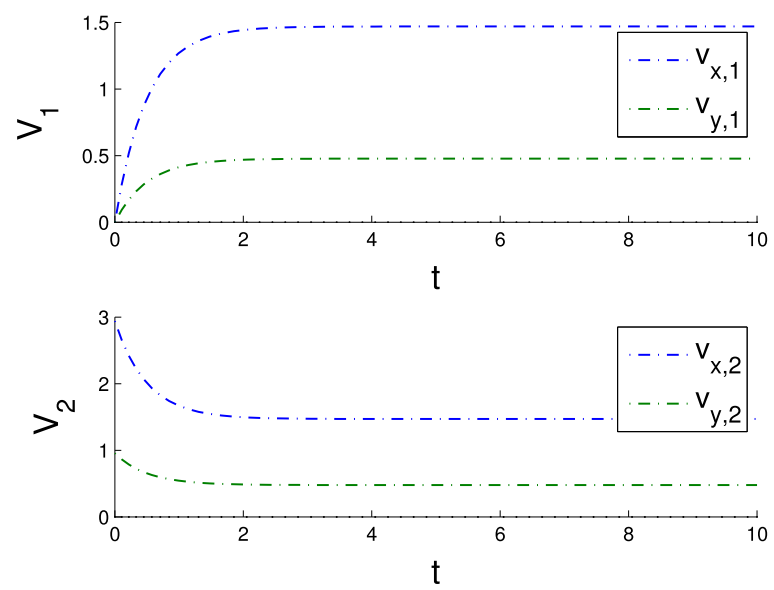

(b) The convergence of absolute velocities of the agents.

Fig. 3. Two agent case with $\mathbf{D} \neq 0$.

Theorem 2. Consider the time-varying mismatched formation control algorithm (5). Suppose that $\phi(t) \in[-\epsilon, \epsilon], \forall t \geq 0$, where $0<\epsilon<\pi$ is a given constant. It follows that

[Intra-formation motion]

(I) The final error between the actual final formation and the desired one $\mathbf{A}_{2}(t)-\mathbf{A}_{1}(t)-\mathbf{D}$ depends on $\|D\|$ and $\epsilon$, independent of the initial states of agents and how $\phi(t)$ is changing. In particular, $\mathbf{A}_{2}(t)-\mathbf{A}_{1}(t)-\mathbf{D}$ is globally ultimately bounded with ultimate bound $\frac{\epsilon}{2 \theta}\|\mathbf{D}\|$, where $0<\theta<1$ is a constant selectable by the designer.

(II) If $\mathbf{D} \neq 0$, the relative velocity of the agents is bounded, but does not have to converge to zero. In particular, $\mathbf{V}_{2}(t)-\mathbf{V}_{1}(t)$ is globally ultimately bounded with ultimate bound $\left(\frac{1}{\theta}+1\right) \epsilon\|\mathbf{D}\|$. If $\mathbf{D}=0$, $\lim _{t \rightarrow \infty}\left(\mathbf{V}_{2}(t)-\mathbf{V}_{1}(t)\right)=0$.

[Whole-formation motion]

(III) If $\mathbf{D} \neq 0$, the absolute velocities of the agents do not converge to zero, but are globally ultimately bounded. If $\mathbf{D}=0$, the absolute velocities of the agents converge to zero exponentially fast.

(IV) If $\mathbf{D} \neq 0$, the agent positions $\mathbf{A}_{1}(t)$ and $\mathbf{A}_{2}(t)$ are neither convergent nor globally ultimately bounded. If $\mathbf{D}=0$, $\lim _{t \rightarrow \infty} \mathbf{A}_{1}(t)=\lim _{t \rightarrow \infty} \mathbf{A}_{2}(t)=\frac{\mathbf{A}_{1}(0)+\mathbf{A}_{2}(0)}{2}$, where $\mathbf{A}_{1}(0)$ and $\mathbf{A}_{2}(0)$ are the initial states of agents 1 and 2 .
Proof. (I) It is not hard to show that (5) can be written as

$\dot{\mathbf{A}}_{1}=\mathbf{A}_{2}-\mathbf{A}_{1}-\mathbf{D}$,
$\dot{\mathbf{A}}_{2}=\mathbf{A}_{1}-\mathbf{A}_{2}+R(\phi(t)) \mathbf{D}$.

Define $\mathbf{Q}(t)=\mathbf{A}_{1}(t)-\mathbf{A}_{2}(t)+\mathbf{D}$. It then follows that

$\dot{\mathbf{Q}}=-2 \mathbf{Q}+(\mathbf{I}-R(\phi(t))) \mathbf{D}$.

Define the Lyapunov candidate $V=\mathbf{Q}^{\mathrm{T}} \mathbf{Q}$ and choose a constant satisfying $0<\theta<1$, where $\theta$ is selectable by the designer and describes the tradeoff between the convergence speed and the ultimate bound. It then follows that

$$
\begin{aligned}
\dot{V}= & -2 \mathbf{Q}^{\mathrm{T}} \mathbf{Q}+\mathbf{Q}(\mathbf{I}-R(\phi(t))) \mathbf{D} \\
= & -2 \theta\|\mathbf{Q}\|^{2}-2(1-\theta)\|\mathbf{Q}\|^{2} \\
& +\mathbf{Q}\left[\begin{array}{cc}
1-\cos \phi(t) & -\sin \phi(t) \\
\sin \phi(t) & 1-\cos \phi(t)
\end{array}\right] \mathbf{D} \\
= & -2 \theta\|\mathbf{Q}\|^{2}-2(1-\theta)\|\mathbf{Q}\|^{2}
\end{aligned}
$$




$$
\begin{aligned}
& \quad+2 \sin \frac{\phi(t)}{2} \mathbf{Q}\left[\begin{array}{cc}
\sin \frac{\phi(t)}{2} & -\cos \frac{\phi(t)}{2} \\
\cos \frac{\phi(t)}{2} & \sin \frac{\phi(t)}{2}
\end{array}\right] \mathbf{D} \\
& \leq-2\|\mathbf{Q}\|\left(\theta\|\mathbf{Q}\|-\left|\sin \frac{\phi(t)}{2}\right|\|\mathbf{D}\|\right)-2(1-\theta)\|\mathbf{Q}\|^{2} \\
& \leq-2\|\mathbf{Q}\|\left(\theta\|\mathbf{Q}\|-\frac{|\phi(t)|}{2}\|\mathbf{D}\|\right)-2(1-\theta)\|\mathbf{Q}\|^{2} \\
& \leq-2\|\mathbf{Q}\|\left(\theta\|\mathbf{Q}\|-\frac{\epsilon}{2}\|\mathbf{D}\|\right)-2(1-\theta)\|\mathbf{Q}\|^{2}, \\
& \text { where we have used the fact that }\left\|\left[\begin{array}{cc}
\sin \frac{\phi(t)}{2} & -\cos \frac{\phi(t)}{2} \\
\cos \frac{\phi(t)}{2} & \sin \frac{\phi(t)}{2}
\end{array}\right]\right\| \leq 1 \text {. It }
\end{aligned}
$$
thus follows that $\dot{V} \leq-2(1-\theta)\|\mathbf{Q}\|^{2}, \forall\|\mathbf{Q}\| \geq \frac{\epsilon}{2 \theta}\|\mathbf{D}\|$. Then, based on Theorem 4.18 of Khalil (2002) (uniform ultimate boundedness theorem), we know that $\mathbf{A}_{2}-\mathbf{A}_{1}-\mathbf{D}$ is globally ultimately bounded with ultimate bound $\frac{\epsilon}{2 \theta}\|\mathbf{D}\|$.

(II) Since $\left\|\mathbf{V}_{2}(t)-\mathbf{V}_{1}(t)\right\|=\|\dot{\mathbf{Q}}(t)\|=\|-2 \mathbf{Q}(t)+(\mathbf{I}-$ $R(\phi(t))) \mathbf{D}\|\leq 2\| \mathbf{Q}(t)\|+\epsilon\| \mathbf{D} \|$, it follows that $\mathbf{V}_{2}(t)-\mathbf{V}_{1}(t)$ is globally ultimately bounded with ultimate bound $\left(\frac{1}{\theta}+1\right) \epsilon\|\mathbf{D}\|$.

From (6) it is straightforward to obtain (III) and (IV).

Remark 2. Instead of solving the explicit solution of (5), Lyapunov analysis approach is used to obtain ultimate boundedness properties for Theorem 2. Therefore, the explicit expression of convergence time $T$ in the form of ultimate bound $\beta$ is not straightforwardly available. However, according to the proof of Theorem 2 , the convergence time is clearly related to $1-\theta$. Therefore, there exists a tradeoff between ultimate bound and convergence speed and this tradeoff is determined by the free parameter $\theta$. More specifically, large ultimate bound corresponds to fast convergence speed while small ultimate bound corresponds to slow convergence speed.

\section{Estimation and compensation algorithms for two agent case}

In this section, we first consider the estimation of $\phi$. We shall show later how the use of an estimate of $\phi$ can eliminate the problem caused by the compass mismatch.

Since each agent in the steady state will perceive a discrepancy in its own coordinate basis between the desired formation and the actual formation, with an angular error of $\frac{\phi}{2}$, each agent is able to estimate $\phi$ separately using its own available information. Without loss of generality, we let agent 2 be responsible for the estimation of $\phi$. The following compensation control algorithm is proposed

${ }^{1} \dot{\mathbf{A}}_{1}=\mathbf{A}_{2}-\mathbf{A}_{1}-\mathbf{D}$,

${ }^{2} \dot{\mathbf{A}}_{2}=R(-\phi)\left(\mathbf{A}_{1}-\mathbf{A}_{2}\right)+\mathbf{D}+\mathbf{U}$,

where $\mathbf{U} \in \mathbb{R}^{2}$ denotes a compensation input. We next specify how to design the estimation of $\phi$ and use the estimated information on $\phi$ to compensate the original mismatched algorithm such that the desired formation is achieved.

\subsection{Estimation of $\phi$ using absolute position information}

In this subsection, we apply Theorem 1 to estimate $\phi$, and therefore consider the case that $\mathbf{U}=0$. We assume that the absolute (as opposed to relative) position information of agent 2 is available for agent 2 and expressed in the coordinate basis of agent 2, i.e., ${ }^{2} \mathbf{A}_{2}=\left[{ }^{2} x_{2},{ }^{2} y_{2}\right]^{\mathrm{T}}$ is available for agent 2 . We also assume that $\mathbf{D} \neq 0$ (the case of $\mathbf{D}=0$ is no longer considered in the subsequent exposition as rendezvous is still achieved for this case even if there exist mismatched compasses).

We propose the following estimator for agent 2 :

$\dot{z}=-z-\frac{{ }^{2} y_{2} d_{x}-{ }^{2} x_{2} d_{y}}{d_{x}^{2}+d_{y}^{2}}, \quad \hat{\phi}= \begin{cases}\frac{\pi}{2}, & \varphi>1 \\ \arcsin \varphi, & \varphi \in[-1,1] \\ -\frac{\pi}{2}, & \varphi<-1,\end{cases}$

where $\varphi=2\left(z+\frac{{ }^{2} y_{2} d_{x}-{ }^{2} x_{2} d_{y}}{d_{x}^{2}+d_{y}^{2}}\right)$. The following result concerns the convergence of the directional error estimate $\hat{\phi}(t)$. Note that the hypothesis of the theorem imposes an a priori but rather large bound on $\phi$. We return to this assumption subsequently.

Theorem 3. Consider the mismatched formation control algorithm (2) and the estimator (9). Suppose that $\phi \in\left[-\frac{\pi}{2}, 0\right) \cup\left(0, \frac{\pi}{2}\right]$ and $\mathbf{D} \neq 0$. It follows that $\lim _{t \rightarrow \infty} \hat{\phi}(t)=\phi$ exponentially fast.

Proof. Based on the (IV) of Theorem 1, we know that $\lim _{t \rightarrow \infty}{ }^{2} \mathbf{V}_{2}(t)=\lim _{t \rightarrow \infty} R(-\phi) \mathbf{V}_{2}(t)=\frac{1}{2}(\mathbf{I}-R(-\phi)) \mathbf{D}=$ $\left[\begin{array}{cc}\frac{1-\cos \phi}{2} & -\frac{\sin \phi}{2} \\ \frac{\sin \phi}{2} & \frac{1-\cos \phi}{2}\end{array}\right] \mathbf{D}$, where ${ }^{2} \mathbf{V}_{2}=\left[{ }^{2} v_{x, 2},{ }^{2} v_{y, 2}\right]$ is the absolute velocity of agent 2 expressed in the coordinate basis of agent 2. Further, convergence is exponential. Therefore, it follows that $\lim _{t \rightarrow \infty} \frac{\mathbf{D}^{\mathrm{T}}\left[\begin{array}{cc}0 & 1 \\ -1 & 0\end{array}\right]^{2} \mathbf{V}_{2}(t)}{\mathbf{D}^{\mathrm{T}} \mathbf{D}}=\frac{\sin \phi}{2}$, where $\frac{\mathbf{D}^{\mathrm{T}}\left[\begin{array}{cc}0 & 1 \\ -1 & 0\end{array}\right]^{2} \mathbf{V}_{2}(t)}{\mathbf{D}^{\mathrm{T}} \mathbf{D}}=$ $\frac{{ }^{2} v_{y, 2}(t) d_{x}-{ }^{2} v_{x, 2}(t) d_{y}}{d_{x}^{2}+d_{y}^{2}}$, and again, convergence is exponential.

In addition, by defining $\sigma=z+\frac{{ }^{2} y_{2}(t) d_{x}-{ }^{2} x_{2}(t) d_{y}}{d_{x}^{2}+d_{y}^{2}}-\frac{\sin \phi}{2}$, it follows from (9) that $\dot{\sigma}=-\sigma+\frac{{ }^{2} v_{y, 2}(t) d_{x}-{ }^{2} v_{x, 2}(t) d_{y}}{d_{x}^{2}+d_{y}^{2}}-\frac{\sin \phi}{2}$. It is evident that the above equation is a linear equation in which the homogeneous part is exponentially stable and the driving term converges to zero exponentially fast. Therefore, it follows from the input-tostate stability property (Khalil, 2002) that $\lim _{t \rightarrow \infty} \sigma(t)=0$ or $\lim _{t \rightarrow \infty}\left(z(t)+\frac{d_{x}^{2} y_{2}(t)-d_{y}^{2} x_{2}(t)}{d_{x}^{2}+d_{y}^{2}}\right)=\frac{\sin \phi}{2}$ exponentially fast. This implies that $\lim _{t \rightarrow \infty} \hat{\phi}(t)=\phi$ exponentially fast.

Remark 3. Estimator (9) does not require agent 2 to measure its own velocity, but only its own position. In many cases, position is more straightforward to obtain than velocity, and less noisy. If one postulates that agent 2 can measure its own velocity, then there is a static estimator of $\phi$ provided by $\hat{\phi}= \begin{cases}\frac{\pi}{2}, & \varphi>1 \\ \arcsin \varphi, & \varphi \in[-1,1] \text { where } \\ -\frac{\pi}{2}, & \varphi<-1,\end{cases}$ $\varphi=2\left(\frac{{ }^{2} v_{y, 2}(t) d_{x}-{ }^{2} v_{x, 2}(t) d_{y}}{d_{x}^{2}+d_{y}^{2}}\right)$

\subsection{Estimation of $\phi$ using relative position information}

In this subsection, we assume that the relative (rather than absolute) position information of agent 2 is available for agent 2 and expressed in the coordinate basis of agent 2, i.e., ${ }^{2} \mathbf{A}_{2}-{ }^{2} \mathbf{A}_{1}=$ $\left[{ }^{2} x_{2}-{ }^{2} x_{1},{ }^{2} y_{2}-{ }^{2} y_{1}\right]^{\mathrm{T}}$ is available for agent 2 .

It follows from (8a) and the fact ${ }^{2} \dot{\mathbf{A}}_{1}=R(-\phi){ }^{1} \dot{\mathbf{A}}_{1}$ that ${ }^{2} \dot{\mathbf{A}}_{1}=$ $R(-\phi)\left(\mathbf{A}_{2}-\mathbf{A}_{1}\right)-R(-\phi) \mathbf{D}$. Therefore, using (8b), we know that ${ }^{2} \dot{\mathbf{A}}_{2}-{ }^{2} \dot{\mathbf{A}}_{1}=-2\left({ }^{2} \mathbf{A}_{2}-{ }^{2} \mathbf{A}_{1}\right)+R(-\phi) \mathbf{D}+\mathbf{D}+\mathbf{U}$. It then follows that for any estimate $\hat{\phi}(t), R(-\phi) \mathbf{D}-R(-\hat{\phi}(t)) \mathbf{D}={ }^{2} \dot{\mathbf{A}}_{2}-{ }^{2} \dot{\mathbf{A}}_{1}$ 
$+2\left({ }^{2} \mathbf{A}_{2}-{ }^{2} \mathbf{A}_{1}\right)-R(-\hat{\phi}(t)) \mathbf{D}-\mathbf{D}-\mathbf{U}$. In the light of this equation, we propose the following estimation algorithm for $\hat{\phi}(t)$ :

$$
\begin{aligned}
\dot{z}= & -k\left(2 d_{x}\left({ }^{2} y_{2}-{ }^{2} y_{1}\right)-2 d_{y}\left({ }^{2} x_{2}-{ }^{2} x_{1}\right)\right. \\
& \left.+\left(d_{x}^{2}+d_{y}^{2}\right) \sin \hat{\phi}-\left(d_{x} u_{y}-d_{y} u_{x}\right)\right), \\
\hat{\phi}= & z-k\left(d_{x}\left({ }^{2} y_{2}-{ }^{2} y_{1}\right)-d_{y}\left({ }^{2} x_{2}-{ }^{2} x_{1}\right)\right),
\end{aligned}
$$

where $k$ is a positive constant and $\mathbf{U}=\left[u_{x}, u_{y}\right]^{\mathrm{T}}$. We next present the following result regarding the convergence of $\hat{\phi}(t)$ using the estimator (10) for the compensation algorithm (8).

Theorem 4. Consider the estimator (10) for the compensation algorithm (8). Suppose that $|\phi|<\frac{\pi}{4}$ and $|\hat{\phi}(0)| \leq \frac{\pi}{4}$. It follows that $\lim _{t \rightarrow \infty} \hat{\phi}(t)=\phi$.

Proof. Denote $\widetilde{\mathbf{D}}=\left[\begin{array}{cc}0 & -1 \\ 1 & 0\end{array}\right]$ D. It follows by an easy direct calculation that $\widetilde{\mathbf{D}}^{\mathrm{T}}(R(-\phi)-R(-\hat{\phi}(t))) \mathbf{D}=(\sin \hat{\phi}-\sin \phi)\|\mathbf{D}\|^{2}$. Also note that (10) can be written as

$$
\begin{aligned}
\dot{z} & =-k \widetilde{\mathbf{D}}^{\mathrm{T}}\left(2\left({ }^{2} \mathbf{A}_{2}-{ }^{2} \mathbf{A}_{1}\right)-R(-\hat{\phi}(t)) \mathbf{D}-\mathbf{D}-\mathbf{U}\right), \\
\hat{\phi}(t) & =z(t)-k \widetilde{\mathbf{D}}^{\mathrm{T}}\left({ }^{2} \mathbf{A}_{2}-{ }^{2} \mathbf{A}_{1}\right) .
\end{aligned}
$$

It follows that $\dot{\hat{\phi}}=-k \widetilde{\mathbf{D}}^{\mathrm{T}}\left({ }^{2} \dot{\mathbf{A}}_{2}-{ }^{2} \dot{\mathbf{A}}_{1}+2\left({ }^{2} \mathbf{A}_{2}-{ }^{2} \mathbf{A}_{1}\right)-R(-\hat{\phi}(t)) \mathbf{D}\right.$ $-\mathbf{D}-\mathbf{U})=-k \tilde{\mathbf{D}}^{\mathrm{T}}(R(-\phi)-R(-\hat{\phi}(t))) \mathbf{D}=-k(\sin \hat{\phi}-$ $\sin \phi)\|\mathbf{D}\|^{2}=-2 k \sin \left(\frac{\hat{\phi}-\phi}{2}\right) \cos \left(\frac{\hat{\phi}+\phi}{2}\right)\|\mathbf{D}\|^{2}$. Choose a Lyapunov function candidate as $V=(\hat{\phi}-\phi)^{2}$. Since $|\hat{\phi}(0)| \leq \frac{\pi}{4}$ and $|\phi|<\frac{\pi}{4}$, it follows that $\cos \left(\frac{\hat{\phi}(0)+\phi}{2}\right)>0$. This implies that $\dot{V} \leq 0$ at $t=0$. We thus know that $\left|\hat{\phi}\left(0^{+}\right)-\phi\right| \leq|\hat{\phi}(0)-\phi|<\frac{\pi}{2}$. Noting that $|\hat{\phi}(t)+\phi|=|\hat{\phi}(t)-\phi+2 \phi|<|\hat{\phi}(t)-\phi|+\frac{\pi}{2}$, it follows that $\left|\hat{\phi}\left(0^{+}\right)+\phi\right|<\pi$ and $\cos \left(\frac{\hat{\phi}\left(0^{+}\right)+\phi}{2}\right)>0$. Therefore, $\dot{V} \leq 0$ at $t=0^{+}$. We next use this observation to show that $|\hat{\phi}(\bar{t})-\phi|<\frac{\pi}{2}$, for all $t \geq 0$ using a contradiction argument. Suppose it is not true. Then, there must exist a finite time instant $T$ for which $|\hat{\phi}(T)-\phi|=\frac{\pi}{2}$ and $|\hat{\phi}(t)-\phi|<\frac{\pi}{2}$ for all $t \in[0, T)$. Based on the continuity, there must exist a neighborhood $[T-\delta, T)$ such that $|\hat{\phi}(t)-\phi|$ is increasing during $t \in[T-\delta, T)$, where $\delta>0$. However, based on the definition of $T$ and the observation for the case of $t=0^{+}$, we know that $\dot{V}(t) \leq 0$, for all $t \in[0, T)$. This shows that $|\hat{\phi}(t)-\phi|$ is non-increasing during $t \in[0, T)$ and therefore indicates a contradiction. Therefore, we know that $\dot{V} \leq 0$, for all $t \geq 0$. Then, based on the Lasalle invariance principle, we can conclude that $\lim _{t \rightarrow \infty} \hat{\phi}(t)=\phi$.

Remark 4. Estimator (9) is used for the case when an absolute position measurement unit is equipped, e.g., GPS while estimator (10) is used for the case when a relative position measurement unit is equipped, e.g., a laser. The design of estimator (10) is similar to a traditional estimator, where the external input to the system whose state is being estimated is normally introduced into the state estimator as well (later in Section 3.3). The convergence of (10) is independent of the time function $\mathbf{U}(t)$. In contrast, an adjustable constant cannot be usefully introduced for (9) in order to separate the time scales for convergence of controller and estimator. The reason is that estimator (9) relies on the convergence of controller (2).

Remark 5. It is reasonable to assume in most cases the validity of an overall bound on $\phi$. Of the different cases giving rise to directional error, probably only long term drift of an inertial navigation system away from an initially correctly calibrated value can give a sizeable error. If the compensation algorithm suggested below is implemented before much drift has occurred, then compensation will be successful.

\subsection{Compensation algorithm}

In this subsection, we show how to use the estimated information on $\phi$ to compensate the original mismatched algorithm such that the desired formation is achieved. Specifically, the following compensation control algorithm is proposed

$\mathbf{U}(t)=-\mathbf{D}+R(-\hat{\phi}(t)) \mathbf{D}$

where $\hat{\phi}(t)$ is obtained by the estimator (10).

Theorem 5. Consider the algorithm (8) with compensation input (11) and the estimator (10). Suppose that $|\phi|<\frac{\pi}{4}$ and $|\hat{\phi}(0)| \leq$ $\frac{\pi}{4}$. Then $\lim _{t \rightarrow \infty} \hat{\phi}(t)=\phi, \lim _{t \rightarrow \infty}\left(\mathbf{A}_{2}(t)-\mathbf{A}_{1}(t)\right)=\mathbf{D}$, and $\lim _{t \rightarrow \infty} \mathbf{V}_{1}(t)=\lim _{t \rightarrow \infty} \mathbf{V}_{2}(t)=0$.

Proof. It is not hard to show from (8) and (11) that $\dot{\mathbf{A}}_{1}-\dot{\mathbf{A}}_{2}=$ $-2\left(\mathbf{A}_{1}-\mathbf{A}_{2}+\mathbf{D}\right)+\mathbf{D}-R(\phi-\hat{\phi}) \mathbf{D}$. Noting that we have shown from Theorem 4 that $\lim _{t \rightarrow \infty} R(\phi-\hat{\phi}(t))=\mathbf{I}$, it then follows from the input-to-state stability property (Khalil, 2002) that $\lim _{t \rightarrow \infty}\left(\mathbf{A}_{2}(t)-\mathbf{A}_{1}(t)\right)=\mathbf{D}$. It is then trivial to show that $\lim _{t \rightarrow \infty} \mathbf{V}_{1}(t)=\lim _{t \rightarrow \infty} \mathbf{V}_{2}(t)=0$.

\section{4. $n$ agent case}

In this section, we aim to show how the analysis of the previous section for two agents will carry over to $n \geq 3$ agents.

Notation. An undirected graph $g$ consists of a pair $(\mathcal{V}, \mathcal{E})$, where $\mathcal{V}=\{1,2, \ldots, n\}$ is a finite, nonempty set of nodes and $\mathcal{E} \subseteq \mathcal{V} \times \mathcal{V}$ is a set of unordered pairs of nodes. An edge $\{j, i\} \in \mathcal{E}$ denotes that nodes $i, j$ can obtain each other's information mutually. The neighbors of node $i$ are denoted by $\mathcal{N}_{i}:=\{j:\{j, i\} \in \mathcal{E}\}$. The adjacency matrix $\widetilde{A}=\left[a_{i j}\right] \in \mathbb{R}^{n \times n}$ associated with the graph $g$ is defined such that $a_{i j}=1$ if $\{j, i\} \in \varepsilon$ and $a_{i j}=0$ otherwise. It is obvious that $a_{i j}=a_{j i}$, for all $i, j \in \mathcal{V}$ for the undirected graph.

We suppose that $n$ agents are denoted by $1,2, \ldots, n$ in a 2-dimensional plane. We assume that there is an underlying connected graph with agents corresponding to vertices, and edges corresponding to specified desired relative positions, which must be consistent with a realizable formation. The classical formation control algorithm for each agent is given by: $\dot{\mathbf{A}}_{i}=\sum_{j \in \mathcal{N}_{i}}\left(\mathbf{A}_{j}-\mathbf{A}_{i}+\right.$ $\left.\mathbf{D}_{i j}\right), i \in \mathcal{V}$, where $\mathbf{A}_{i}=\left[x_{i}, y_{i}\right]^{\mathrm{T}} \in \mathbb{R}^{2}, \forall i \in \mathcal{V}, \mathbf{D}_{i j}=\left[d_{x, i j}, d_{y, i j}\right]^{\mathrm{T}} \in$ $\mathbb{R}^{2}, \forall i, j \in \mathcal{V}$ are given constants defining the specified relative positions and known for each agent, $\mathcal{N}_{i}, i \in \mathcal{V}$ denotes the neighbor set of agent $i$. It is obvious that $\mathbf{D}_{i i}=0$, and $\mathbf{D}_{i j}=-\mathbf{D}_{j i}, \forall i, j \in \mathcal{V}$, and we assume that the graph is connected, the neighbor sets $\mathcal{N}_{i}$ together with $\mathbf{D}_{i j}$ are properly defined in order that the above algorithm will ensure convergence to a well-defined and unique formation with the desired specified relative positions. Now the full set $\mathbf{D}_{1 j}, j=2, \ldots, n$, may not appear in the list of desired relative positions. Nevertheless, the values that all these relative positions will assume in a correct formation are easily computable from the data. Conversely, given all the specified desired relative positions, any other relative position is computable from them. Accordingly, an equivalent objective to the original one is to ensure that asymptotically as $t \rightarrow \infty$ one has $\mathbf{A}_{1}(t)-\mathbf{A}_{2}(t)=\mathbf{D}_{12}$, $\mathbf{A}_{1}(t)-\mathbf{A}_{3}(t)=\mathbf{D}_{13}, \ldots$, and $\mathbf{A}_{1}(t)-\mathbf{A}_{n}(t)=\mathbf{D}_{1 n}$. We define $\mathbf{D}=\left[\mathbf{D}_{12}^{\mathrm{T}}, \mathbf{D}_{13}^{\mathrm{T}}, \ldots, \mathbf{D}_{1 n}^{\mathrm{T}}\right]^{\mathrm{T}}$. Due to the existence of mismatched compasses, and expressing measured multi-agent distances using 
the coordinate basis associated with agent 1 (without loss of generality), the actual formation control algorithm becomes:

$\begin{aligned}{ }^{1} \dot{\mathbf{A}}_{1} & =\sum_{j \in \mathcal{N}_{1}}\left(\mathbf{A}_{j}-\mathbf{A}_{1}+\mathbf{D}_{1 j}\right) \\ { }^{2} \dot{\mathbf{A}}_{2} & =\sum_{j \in \mathcal{N}_{2}}\left(R\left(-\phi_{2}\right)\left(\mathbf{A}_{j}-\mathbf{A}_{2}\right)+\mathbf{D}_{2 j}\right)\end{aligned}$

${ }^{n} \dot{\mathbf{A}}_{n}=\sum_{j \in \mathcal{N}_{n}}\left(R\left(-\phi_{n}\right)\left(\mathbf{A}_{j}-\mathbf{A}_{n}\right)+\mathbf{D}_{n j}\right)$,

where $\phi_{2}, \phi_{3}, \ldots, \phi_{n} \in(-\pi, \pi]$ denote the angular mismatch between the coordinate axes of agent 1 and agent $i, \forall i=$ $2,3, \ldots, n, R\left(-\phi_{i}\right)=\left[\begin{array}{cc}\cos \phi_{i} & \sin \phi_{i} \\ -\sin \phi_{i} & \cos \phi_{i}\end{array}\right], \forall i=2,3, \ldots, n$.

It is not hard to show that (12) can be written as

$\dot{\mathbf{A}}_{1}=\sum_{j \in \mathcal{N}_{1}}\left(\mathbf{A}_{j}-\mathbf{A}_{1}+\mathbf{D}_{1 j}\right)$
$\dot{\mathbf{A}}_{2}=\sum_{j \in \mathcal{N}_{2}}\left(\mathbf{A}_{j}-\mathbf{A}_{2}+R\left(\phi_{2}\right) \mathbf{D}_{2 j}\right)$

$\dot{\mathbf{A}}_{n}=\sum_{j \in \mathcal{N}_{n}}\left(\mathbf{A}_{j}-\mathbf{A}_{n}+R\left(\phi_{n}\right) \mathbf{D}_{n j}\right)$

We next establish the following result for the case of $n$ agents with a general connected graph.

Theorem 6. Consider the mismatched formation control algorithm (12). Suppose that $\phi_{2}, \phi_{3}, \ldots, \phi_{n} \neq 0$ with all $\phi$ constant for all time. It follows that

[Intra-formation motion]

(I) The agents converge to a fixed formation exponentially fast.

(II) The relative velocity of each agent pair converges to zero exponentially fast, i.e., $\lim _{t \rightarrow \infty}\left(\mathbf{V}_{i}(t)-\mathbf{V}_{j}(t)\right)=0$, for all $i, j \in \mathcal{V}$.

(III) If $\mathbf{D} \neq 0$, the final formation is distorted from the desired one and the final formation distortion is $O\left(\max _{j=2,3, \ldots, n}\left|\phi_{j}\right|\right)$, as $\phi_{2}, \phi_{3}, \ldots, \phi_{n} \rightarrow 0$. If $\mathbf{D}=0, \lim _{t \rightarrow \infty}\left(\mathbf{A}_{i}(t)-\mathbf{A}_{j}(t)\right)=0$, for all $i, j \in \mathcal{V}$.

[Whole-formation motion]

(IV) If $\mathbf{D} \neq 0$, the absolute velocity of each agent converges to the same nonzero constant exponentially fast. In particular, $\lim _{t \rightarrow \infty}\left\|\mathbf{V}_{i}(t)\right\|=O\left(\max _{j=2,3, \ldots, n}\left|\phi_{j}\right|\right)$, as $\phi_{2}, \phi_{3}, \ldots, \phi_{n} \rightarrow$ 0 , for all $i \in \mathcal{V}$.

(V) If $\mathbf{D} \neq 0$, the agent position $\mathbf{A}_{i}(t), \forall i \in \mathcal{V}$ is neither convergent nor bounded. If $\mathbf{D}=0, \lim _{t \rightarrow \infty} \mathbf{A}_{1}(t)=\cdots=$ $\lim _{t \rightarrow \infty} \mathbf{A}_{n}(t)=\frac{\mathbf{A}_{1}(0)+\cdots+\mathbf{A}_{n}(0)}{n}$, where $\mathbf{A}_{1}(0), \mathbf{A}_{2}(0), \ldots, \mathbf{A}_{n}(0)$ are the initial states of agents $1,2, \ldots, n$.

Proof. Using the relations that $\mathbf{A}_{j}-\mathbf{A}_{k}=\left(\mathbf{A}_{j}-\mathbf{A}_{1}\right)+\left(\mathbf{A}_{1}-\mathbf{A}_{k}\right)$ and $\mathbf{D}_{k j}=\mathbf{D}_{1 j}-\mathbf{D}_{1 k}, \forall j, k \in \mathcal{V}$, (12) can be written as

$\dot{\mathbf{A}}_{1}=\sum_{j \in \mathcal{N}_{1}}\left(\mathbf{A}_{j}-\mathbf{A}_{1}+\mathbf{D}_{1 j}\right)$

$\dot{\mathbf{A}}_{n}=N_{n}\left(\mathbf{A}_{1}-\mathbf{A}_{n}\right)-N_{n} R\left(\phi_{n}\right) \mathbf{D}_{1 n}+\sum_{j \in \mathcal{N}_{n}}\left(\mathbf{A}_{j}-\mathbf{A}_{1}+R\left(\phi_{n}\right) \mathbf{D}_{1 j}\right)$,

where $N_{i}$ denotes the cardinality of set $\mathcal{N}_{i}, i \in \mathcal{V}$.
Therefore, the intra-formation motion can be written in the compact form as: $\dot{\mathbf{Q}}=-P \mathbf{Q}$, where $\mathbf{Q}=\left[\begin{array}{c}\mathbf{A}_{1}-\mathbf{A}_{2} \\ \mathbf{A}_{1}-\mathbf{A}_{3} \\ \vdots \\ \mathbf{A}_{1}-\mathbf{A}_{n}\end{array}\right]-P^{-1} W\left[\begin{array}{c}\mathbf{D}_{12} \\ \mathbf{D}_{13} \\ \vdots \\ \mathbf{D}_{1 n}\end{array}\right]$, $P=\left[\begin{array}{cccc}N_{2} & 0 & \ldots & 0 \\ 0 & N_{3} & & \vdots \\ \vdots & & \ddots & 0 \\ 0 & \ldots & 0 & N_{n}\end{array}\right] \otimes \mathbf{I}+\left[\begin{array}{ccc}a_{12} & a_{13} & \ldots \\ a_{12} & a_{13} & \cdots \\ \vdots & & \\ a_{12} & a_{13} & \ldots\end{array}\right] \otimes \mathbf{I}-$ $\left[\begin{array}{cccc}0 & a_{23} & a_{24} & \cdots \\ a_{32} & 0 & a_{34} & \cdots \\ \vdots & & \ddots & \vdots \\ a_{n 2} & a_{n 3} & \cdots & 0\end{array}\right] \otimes \mathbf{I}, W=\left[\begin{array}{cccc}N_{2} R\left(\phi_{2}\right) & 0 & \cdots & 0 \\ 0 & N_{3} R\left(\phi_{3}\right) & & \vdots \\ \vdots & & \ddots & 0 \\ 0 & \ldots & 0 & N_{n} R\left(\phi_{n}\right)\end{array}\right]+$
$\left[\begin{array}{ccc}a_{12} & a_{13} & \ldots \\ a_{12} & a_{13} & \cdots \\ \vdots & & \\ a_{12} & a_{13} & \cdots\end{array}\right] \otimes \mathbf{I}-\left[\begin{array}{cccc}0 & a_{23} R\left(\phi_{2}\right) & a_{24} R\left(\phi_{2}\right) & \cdots \\ a_{32} R\left(\phi_{3}\right) & 0 & a_{34} R\left(\phi_{3}\right) & \cdots \\ \vdots & & \ddots & \vdots \\ a_{n 2} R\left(\phi_{n}\right) & a_{n 3} R\left(\phi_{n}\right) & \ldots & 0\end{array}\right]$, and $a_{i j}$ denotes $(i, j)$ th entry of the adjacency matrix $\ddot{\widetilde{A}}$. Based on the transformation and Lemma 1 given in Peng and Yang (2009), it is not hard to show that $-P$ is a Hurwitz stable matrix when the underlying graph is connected. Therefore, $\lim _{t \rightarrow \infty} \mathbf{Q}(t)=0$.

(II) It follows from (I) that $\lim _{t \rightarrow \infty}\left(\dot{\mathbf{A}}_{1}(t)-\dot{\mathbf{A}}_{j}(t)\right)=0$, for all $j=2,3, \ldots, n$.

(III) For the case of $\mathbf{D} \neq 0$, we know that the actual final formation is determined by $\lim _{t \rightarrow \infty}\left[\begin{array}{c}\mathbf{A}_{1}(t)-\mathbf{A}_{2}(t) \\ \mathbf{A}_{1}(t)-\mathbf{A}_{3}(t) \\ \vdots \\ \mathbf{A}_{1}(t)-\mathbf{A}_{n}(t)\end{array}\right]=P^{-1} W \mathbf{D}$. However, the desired formation is determined by $\mathbf{D}$. The final formation distortion $^{1}$ is defined and given by $\delta \mathbf{D}:=\left\|P^{-1} W \mathbf{D}-\mathbf{D}\right\|$. It then follows that

$$
\begin{aligned}
\delta \mathbf{D}= & \left\|P^{-1} \Omega \mathbf{D}\right\| \leq c_{1}\left\|\Omega_{1}\right\|\|\mathbf{D}\| \\
\leq & c_{1} \times \sqrt{2(n-1)} \times 2 \\
& \times \max _{j=2,3, \ldots, n}\left\{\left(N_{j}+\sum_{k=2}^{n} a_{j k}\right)\left|\sin \frac{\phi_{j}}{2}\right|\left(\left|\sin \frac{\phi_{j}}{2}\right|+\left|\cos \frac{\phi_{j}}{2}\right|\right)\right\}\|\mathbf{D}\| \\
\leq & c_{1} \times \sqrt{2(n-1)} \times 2 \times 4 n \times \max _{j=2,3, \ldots, n}\left|\sin \frac{\phi_{j}}{2}\right|\|\mathbf{D}\| \\
\leq & 4 c_{1} n \sqrt{2(n-1)}\|\mathbf{D}\| \max _{j=2,3, \ldots, n}\left|\phi_{j}\right|,
\end{aligned}
$$

where $\Omega$ and $\Omega_{1}$ are given in (18) and (19), respectively (see Box I). $c_{1}=\left\|P^{-1}\right\|$ is a positive constant and we have used the facts that $R\left(\phi_{j}\right)-\mathbf{I}=-2 \sin \frac{\phi_{j}}{2}\left[\begin{array}{cr}\sin \frac{\phi_{j}}{2} & \cos \frac{\phi_{j}}{2} \\ -\cos \frac{\phi_{j}}{2} & \sin \frac{\phi_{j}}{2}\end{array}\right], j \in\{2,3, \ldots, n\}$ for the first inequality, $\|A\|_{2} \leq \sqrt{m}\|A\|_{\infty}=\sqrt{m} \max _{1 \leq i \leq m} \sum_{j=1}^{l}\left|a_{i j}\right|$ for a matrix $\mathbb{R}^{m \times l},|\sin \alpha| \leq 1$ and $|\cos \alpha| \leq 1, \forall \alpha \in \mathbb{R}$ for the second inequality. Therefore, $\delta \mathbf{D}=O\left(\max _{j=2,3, \ldots, n}\left|\phi_{j}\right|\right)$, as $\phi_{2}, \phi_{3}, \ldots, \phi_{n} \rightarrow 0$. In addition, it is trivial to prove the case of $\mathbf{D}=0$.

\footnotetext{
1 Of course, we could choose to define the distortion using all interagent distances and not just those occurring in the tree. The bound will be $(n-1) \sqrt{n}$ times the bound computed here.
} 


$$
\begin{aligned}
& \Omega=\left[\begin{array}{cccc}
N_{2}\left(R\left(\phi_{2}\right)-\mathbf{I}\right) & a_{23}\left(\mathbf{I}-R\left(\phi_{2}\right)\right) & a_{24}\left(\mathbf{I}-R\left(\phi_{2}\right)\right) & \ldots \\
a_{32}\left(\mathbf{I}-R\left(\phi_{3}\right)\right) & N_{3}\left(R\left(\phi_{3}\right)-\mathbf{I}\right) & a_{34}\left(\mathbf{I}-R\left(\phi_{3}\right)\right) & \ldots \\
\vdots & & \ddots & \vdots \\
a_{n 2}\left(\mathbf{I}-R\left(\phi_{n}\right)\right) & a_{n 3}\left(\mathbf{I}-R\left(\phi_{n}\right)\right) & \ldots & N_{n}\left(R\left(\phi_{n}\right)-\mathbf{I}\right)
\end{array}\right]
\end{aligned}
$$

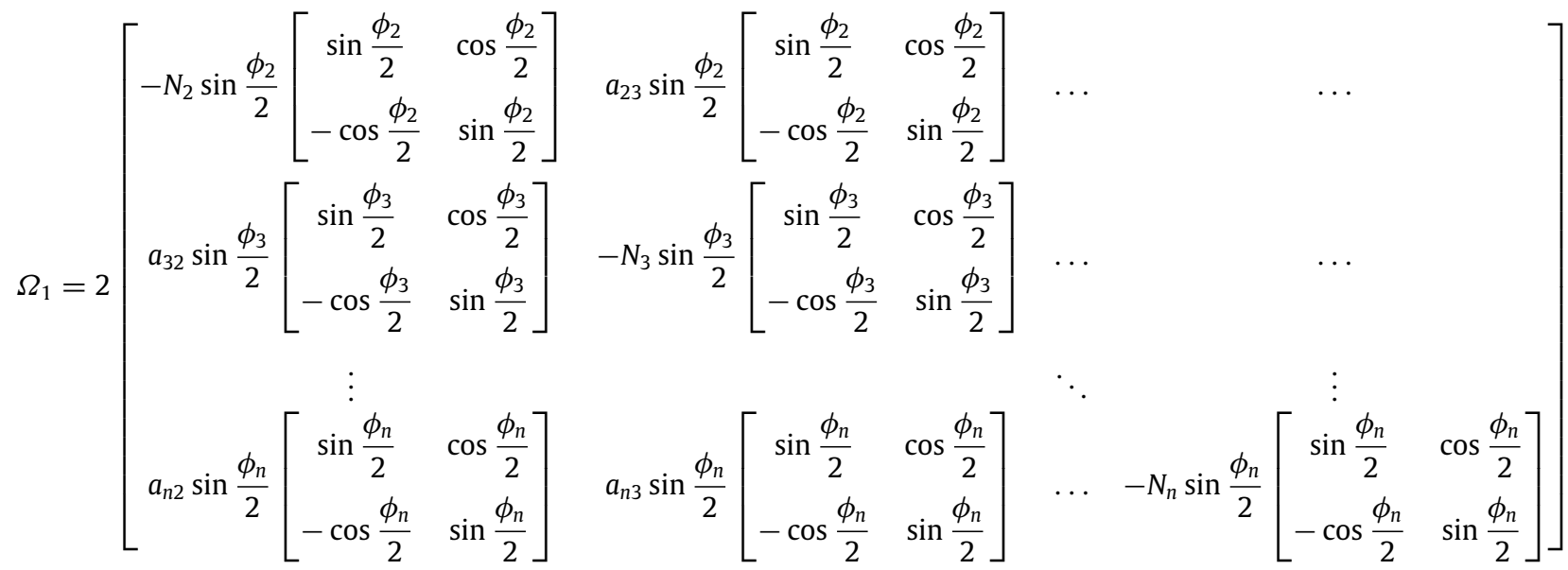

Box I.

(IV) For the case of $\mathbf{D} \neq 0$, it follows from (13) that

$$
\begin{aligned}
\dot{\mathbf{A}}_{1}(t)= & \sum_{j \in \mathcal{N}_{1}}\left(\mathbf{A}_{j}-\mathbf{A}_{1}+\mathbf{D}_{1 j}\right)=\left[\begin{array}{llll}
-a_{12} & -a_{13} & \ldots & -a_{1 n}
\end{array}\right] \\
& \times\left[\begin{array}{c}
\mathbf{A}_{1}(t)-\mathbf{A}_{2}(t) \\
\mathbf{A}_{1}(t)-\mathbf{A}_{3}(t) \\
\vdots \\
\mathbf{A}_{1}(t)-\mathbf{A}_{n}(t)
\end{array}\right]+\left[\begin{array}{llll}
a_{12} & a_{13} & \ldots & a_{1 n}
\end{array}\right] \mathbf{D} .
\end{aligned}
$$

Therefore, $\lim _{t \rightarrow \infty} \dot{\mathbf{A}}_{1}(t)=\left[\begin{array}{llll}-a_{12} & -a_{13} & \ldots & -a_{1 n}\end{array}\right] \times P^{-1}$ $(W-P) \mathbf{D}=\left[\begin{array}{lll}-\mathbf{I} & \ldots & -\mathbf{I}\end{array}\right] P^{-1} \Omega \mathbf{D}$. This shows that

$\lim _{t \rightarrow \infty}\left\|\dot{\mathbf{A}}_{1}(t)\right\| \leq c_{2}\|\mathbf{D}\| \max _{j=2,3, \ldots, n}\left|\phi_{j}\right|$,

where $c_{2}$ is a positive constant.

Therefore, the absolute velocities obey $\lim _{t \rightarrow \infty}\left\|\dot{\mathbf{A}}_{1}(t)\right\|=$ $\cdots=\lim _{t \rightarrow \infty}\left\|\dot{\mathbf{A}}_{n}(t)\right\|=O\left(\max _{j=2,3, \ldots, n}\left|\phi_{j}\right|\right)$, as $\phi_{2}, \phi_{3}, \ldots, \phi_{n} \rightarrow$ 0 . In addition, it is trivial to prove the claim for the case of $\mathbf{D}=0$. Based on (IV), (V) is obvious.

Remark 6. Theorem 6 shows that the formation distortion and steady velocity of the formation can be bounded using the maximum of all the mismatch angles. In addition, this fact holds for arbitrarily large but finite network size and for an arbitrary connected graph.

\section{Estimation and compensation algorithms for $\boldsymbol{n}$ agent case}

Without loss of generality, we let agent $2,3, \ldots, n$ be responsible for the estimation of $\phi_{2}, \phi_{3}, \ldots, \phi_{n}$, respectively. The following compensation control algorithm is proposed

$$
\begin{aligned}
{ }^{1} \dot{\mathbf{A}}_{1} & =\sum_{j \in \mathcal{N}_{1}}\left(\mathbf{A}_{j}-\mathbf{A}_{1}+\mathbf{D}_{1 j}\right), \\
{ }^{2} \dot{\mathbf{A}}_{2} & =\sum_{j \in \mathcal{N}_{2}}\left(R\left(-\phi_{2}\right)\left(\mathbf{A}_{j}-\mathbf{A}_{2}\right)+\mathbf{D}_{2 j}\right)+\mathbf{U}_{2},
\end{aligned}
$$

$$
{ }^{n} \dot{\mathbf{A}}_{n}=\sum_{j \in \mathcal{N}_{n}}\left(R\left(-\phi_{n}\right)\left(\mathbf{A}_{j}-\mathbf{A}_{n}\right)+\mathbf{D}_{n j}\right)+\mathbf{U}_{n},
$$

where $\mathbf{U}_{2}, \mathbf{U}_{3}, \ldots, \mathbf{U}_{n} \in \mathbb{R}^{2}$ denote compensation inputs. We next specify how to design the estimations of $\phi_{2}, \phi_{3}, \ldots, \phi_{n}$ and use the estimated information on $\phi_{2}, \phi_{3}, \ldots, \phi_{n}$ to compensate the original mismatched algorithm such that the desired formation is achieved.

\subsection{Estimations using relative position information}

In this subsection, we assume that each agent has access to the relative position information respect to all the other agents and the relative position information is expressed in its own coordinate basis, i.e., ${ }^{2} \mathbf{A}_{2}-{ }^{2} \mathbf{A}_{1},{ }^{2} \mathbf{A}_{2}-{ }^{2} \mathbf{A}_{3}, \ldots,{ }^{2} \mathbf{A}_{2}-{ }^{2} \mathbf{A}_{n}$, is available for agent 2; ${ }^{3} \mathbf{A}_{3}-{ }^{3} \mathbf{A}_{1},{ }^{3} \mathbf{A}_{3}-{ }^{3} \mathbf{A}_{2}, \ldots,{ }^{3} \mathbf{A}_{3}-{ }^{3} \mathbf{A}_{n}$, is available for agent 3 ; and the like, where ${ }^{k} \mathbf{A}_{p}-{ }^{k} \mathbf{A}_{q}=\left[{ }^{k} x_{p}-{ }^{k} x_{q},{ }^{k} y_{p}-{ }^{k} y_{q}\right]^{\mathrm{T}}$, for all $k=2,3, \ldots, n$ and $p \neq q \in \mathcal{V}$.

Remark 7. Due to the structure of the proposed estimator below, each agent needs to know the relative position information respect to all the other agents. Therefore, the estimation problem is solved for the $n$ agents with a complete graph in this subsection. The solution for a general connected graph is an open problem and deserves further investigation.

Motivated by (10), we propose the following estimation algorithm for obtaining $\hat{\phi}_{2}(t), \hat{\phi}_{3}(t), \ldots, \hat{\phi}_{n}(t)$ :

$$
\begin{aligned}
\dot{z}_{i}= & \left.-k_{i}\left(N_{1} \hat{d}_{x, i}{ }^{i} y_{i}-{ }^{i} y_{1}\right)-N_{1} \hat{d}_{y, i}{ }^{i} x_{i}-{ }^{i} x_{1}\right) \\
& +\sum_{j \in \mathcal{N}_{i}}\left(\hat{d}_{y, i}\left({ }^{i} x_{j}-{ }^{i} x_{i}\right)-\hat{d}_{x, i}\left({ }^{i} y_{j}-{ }^{i} y_{i}\right)+\hat{d}_{y, i} d_{x, i j}-\hat{d}_{x, i} d_{y, i j}\right) \\
& +\sum_{j \in \mathcal{N}_{1}}\left(\hat{d}_{x, i}\left({ }^{i} y_{j}-{ }^{i} y_{i}\right)-\hat{d}_{y, i}\left({ }^{i} x_{j}-{ }^{i} x_{i}\right)\right) \\
& \left.+\left(\hat{d}_{x, i}^{2}+\hat{d}_{y, i}^{2}\right) \sin \hat{\phi}_{i}-\left(\hat{d}_{x, i} u_{y, i}-\hat{d}_{y, i} u_{x, i}\right)\right) \\
\hat{\phi}_{i}= & z_{i}-k_{i}\left(\hat{d}_{x, i}\left({ }^{i} y_{i}-{ }^{i} y_{1}\right)-\hat{d}_{y, i}\left({ }^{i} x_{i}-{ }^{i} x_{1}\right)\right) \\
i= & 2,3 \ldots, n,
\end{aligned}
$$


where $k_{i}$ is a positive constant, $\mathbf{U}_{i}=\left[u_{x, i}, u_{y, i}\right]^{\mathrm{T}}, \widehat{\mathbf{D}}_{i}=\left(N_{1} \mathbf{D}_{i 1}\right.$ $\left.-\sum_{j \in \mathcal{N}_{1}} \mathbf{D}_{i j}\right), \widehat{\mathbf{D}}_{i}=\left[\hat{d}_{x, i}, \hat{d}_{y, i}\right]^{\mathrm{T}}, \forall i=2,3, \ldots, n$. We next present the following result regarding the convergence of $\hat{\phi}_{2}(t), \hat{\phi}_{3}(t)$, $\ldots, \hat{\phi}_{n}(t)$ using the estimator (15) for the compensation algorithm (14).

Theorem 7. Consider the estimator (15) for the compensation algorithm (14). Suppose that $\left|\phi_{i}\right|<\frac{\pi}{4}$ and $\left|\hat{\phi}_{i}(0)\right| \leq \frac{\pi}{4}$, for all $i=$ $2,3, \ldots, n$. It follows that $\lim _{t \rightarrow \infty} \hat{\phi}_{i}(t)=\phi_{i}$, for all $i=2,3, \ldots, n$.

Proof. It follows from (12a) and the fact ${ }^{2} \dot{\mathbf{A}}_{1}=R\left(-\phi_{2}\right)^{1} \dot{\mathbf{A}}_{1}$ that

${ }^{2} \dot{\mathbf{A}}_{1}=\sum_{j \in \mathcal{N}_{1}}\left(R\left(-\phi_{2}\right)\left(\mathbf{A}_{j}-\mathbf{A}_{1}\right)+R\left(-\phi_{2}\right) \mathbf{D}_{1 j}\right)$.

Therefore, using (12b) and the facts that $\mathbf{A}_{j}-\mathbf{A}_{1}=\left(\mathbf{A}_{j}-\mathbf{A}_{2}\right)+$ $\left(\mathbf{A}_{2}-\mathbf{A}_{1}\right)$ and $\mathbf{D}_{1 j}=\mathbf{D}_{2 j}-\mathbf{D}_{21}, \forall j \in \mathcal{V} \backslash\{1\}$, we know that

$$
\begin{aligned}
{ }^{2} \dot{\mathbf{A}}_{2}-{ }^{2} \dot{\mathbf{A}}_{1}= & -N_{1}\left({ }^{2} \mathbf{A}_{2}-{ }^{2} \mathbf{A}_{1}\right)+R\left(-\phi_{2}\right)\left(N_{1} \mathbf{D}_{21}-\sum_{j \in \mathcal{N}_{1}} \mathbf{D}_{2 j}\right) \\
& +\sum_{j \in \mathcal{N}_{2}}\left({ }^{2} \mathbf{A}_{j}-{ }^{2} \mathbf{A}_{2}+\mathbf{D}_{2 j}\right)-\sum_{j \in \mathcal{N}_{1}}\left({ }^{2} \mathbf{A}_{j}-{ }^{2} \mathbf{A}_{2}\right)+\mathbf{U}_{2} .
\end{aligned}
$$

Similarly, it follows that

$$
\begin{aligned}
{ }^{3} \dot{\mathbf{A}}_{3}-{ }^{3} \dot{\mathbf{A}}_{1}= & -N_{1}\left({ }^{3} \mathbf{A}_{3}-{ }^{3} \mathbf{A}_{1}\right)+R\left(-\phi_{3}\right)\left(N_{1} \mathbf{D}_{31}-\sum_{j \in \mathcal{N}_{1}} \mathbf{D}_{3 j}\right) \\
& +\sum_{j \in \mathcal{N}_{3}}\left({ }^{3} \mathbf{A}_{j}-{ }^{3} \mathbf{A}_{3}+\mathbf{D}_{3 j}\right)-\sum_{j \in \mathcal{N}_{1}}\left({ }^{3} \mathbf{A}_{j}-{ }^{3} \mathbf{A}_{3}\right)+\mathbf{U}_{3}, \\
\vdots & \\
{ }^{n} \dot{\mathbf{A}}_{n}-{ }^{n} \dot{\mathbf{A}}_{1}= & -N_{1}\left({ }^{n} \mathbf{A}_{n}-{ }^{n} \mathbf{A}_{1}\right)+R\left(-\phi_{n}\right)\left(N_{1} \mathbf{D}_{n 1}-\sum_{j \in \mathcal{N}_{1}} \mathbf{D}_{n j}\right) \\
& +\sum_{j \in \mathcal{N}_{n}}\left({ }^{n} \mathbf{A}_{j}-{ }^{n} \mathbf{A}_{n}+\mathbf{D}_{n j}\right)-\sum_{j \in \mathcal{N}_{1}}\left({ }^{n} \mathbf{A}_{j}-{ }^{n} \mathbf{A}_{n}\right)+\mathbf{U}_{n} .
\end{aligned}
$$

Without loss of generality, we next only focus on the analysis of $\hat{\phi}_{2}$. Denote $\widetilde{\mathbf{D}}_{2}=\left[\begin{array}{cc}0 & -1 \\ 1 & 0\end{array}\right] \widehat{\mathbf{D}}_{2}$, where $\widehat{\mathbf{D}}_{2}=\left(N_{1} \mathbf{D}_{21}-\sum_{j \in \mathcal{N}_{1}} \mathbf{D}_{2 j}\right)$. For the case of $i=2$, estimator (15) can be written as

$$
\begin{aligned}
\dot{z}_{2}= & -k_{2} \widetilde{\mathbf{D}}_{2}^{\mathrm{T}}\left(N_{1}\left({ }^{2} \mathbf{A}_{2}-{ }^{2} \mathbf{A}_{1}\right)-R\left(-\hat{\phi}_{2}\right) \widehat{\mathbf{D}}_{2}\right. \\
& \left.-\sum_{j \in \mathcal{N}_{2}}\left({ }^{2} \mathbf{A}_{j}-{ }^{2} \mathbf{A}_{2}+\mathbf{D}_{2 j}\right)+\sum_{j \in \mathcal{N}_{1}}\left({ }^{2} \mathbf{A}_{j}-{ }^{2} \mathbf{A}_{2}\right)-\mathbf{U}_{2}\right), \\
\hat{\phi}_{2} & =z_{2}-k \widetilde{\mathbf{D}}_{2}^{\mathrm{T}}\left({ }^{2} \mathbf{A}_{2}-{ }^{2} \mathbf{A}_{1}\right) .
\end{aligned}
$$

It then follows from direct calculation and (16) that

$$
\begin{aligned}
\dot{\hat{\phi}}= & -k_{2} \widetilde{\mathbf{D}}_{2}^{\mathrm{T}}\left({ }^{2} \dot{\mathbf{A}}_{2}-{ }^{2} \dot{\mathbf{A}}_{1}+N_{1}\left({ }^{2} \mathbf{A}_{2}-{ }^{2} \mathbf{A}_{1}\right)-R\left(-\hat{\phi}_{2}\right) \widehat{\mathbf{D}}_{2}\right. \\
& \left.-\sum_{j \in \mathcal{N}_{2}}\left({ }^{2} \mathbf{A}_{j}-{ }^{2} \mathbf{A}_{2}+\mathbf{D}_{2 j}\right)+\sum_{j \in \mathcal{N}_{1}}\left({ }^{2} \mathbf{A}_{j}-{ }^{2} \mathbf{A}_{2}\right)-\mathbf{U}_{2}\right) \\
= & -k_{2} \widetilde{\mathbf{D}}_{2}^{\mathrm{T}}\left(R\left(-\phi_{2}(t)\right) \widehat{\mathbf{D}}_{2}-R\left(-\hat{\phi}_{2}(t)\right) \widehat{\mathbf{D}}_{2}\right) .
\end{aligned}
$$

It follows from the fact $\widetilde{\mathbf{D}}_{2}^{\mathrm{T}}\left(R\left(-\phi_{2}\right)-R\left(-\hat{\phi}_{2}\right)\right) \widehat{\mathbf{D}}_{2}=\left(\sin \hat{\phi}_{2}-\right.$ $\left.\sin \phi_{2}\right)\left\|\widehat{\mathbf{D}}_{2}\right\|^{2}$ that

$$
\begin{aligned}
\dot{\hat{\phi}} & =-k_{2} \widetilde{\mathbf{D}}_{2}^{\mathrm{T}}\left(R\left(-\phi_{2}\right)-R\left(-\hat{\phi}_{2}\right)\right) \widehat{\mathbf{D}}_{2} \\
& =-k_{2}\left(\sin \hat{\phi}_{2}-\sin \phi_{2}\right)\left\|\widehat{\mathbf{D}}_{2}\right\|^{2} \\
& =-2 k_{2} \sin \left(\frac{\hat{\phi}_{2}-\phi_{2}}{2}\right) \cos \left(\frac{\hat{\phi}_{2}+\phi_{2}}{2}\right)\left\|\widehat{\mathbf{D}}_{2}\right\|^{2} .
\end{aligned}
$$

We thus know from the same analysis as in Theorem 4 that $\lim _{t \rightarrow \infty} \hat{\phi}_{2}(t)=\phi_{2}$. Similarly, it is not hard to show that $\lim _{t \rightarrow \infty} \hat{\phi}_{j}(t)=\phi_{j}$, for all $j=3,4, \ldots, n$.

\subsection{Compensation algorithm}

In this subsection, we show how to use the estimated information on $\phi_{2}, \phi_{3}, \ldots, \phi_{n}$ to compensate the original mismatched algorithm such that the desired formation is achieved. Specifically, the following compensation control algorithm is proposed for all $i=2,3, \ldots, n$,

$\mathbf{U}_{i}=\sum_{j \in \mathcal{N}_{i}}\left(-\mathbf{D}_{i j}+R\left(-\hat{\phi}_{i}\right) \mathbf{D}_{i j}\right)$

where $\hat{\phi}_{i}(t)$ is obtained by the estimator (15).

We next show that $\mathbf{A}_{1}(t)-\mathbf{A}_{i}(t), i=2,3, \ldots, n$, converges to the desired formation using compensation input (17) and the estimator (15).

Theorem 8. Consider the algorithm (14) with compensation input (17) and the estimator (15). Suppose that $\left|\phi_{i}\right|<\frac{\pi}{4}$ and $\left|\hat{\phi}_{i}(0)\right| \leq$ $\frac{\pi}{4}$, for all $i=2,3, \ldots, n$. Then $\lim _{t \rightarrow \infty} \hat{\phi}_{i}(t)=\phi_{i}, \lim _{t \rightarrow \infty}\left(\mathbf{A}_{1}(t)-\right.$ $\left.\mathbf{A}_{i}(t)\right)=\mathbf{D}_{1 i}$, for all $i=2,3, \ldots, n$, and $\lim _{t \rightarrow \infty} \mathbf{V}_{i}(t)=0$, for all $i \in \mathcal{V}$.

Proof. It is not hard to show from (14) and (17) that for all $i=$ $2,3, \ldots, n$,

$$
\begin{aligned}
\dot{\mathbf{A}}_{i}-\dot{\mathbf{A}}_{1}= & -\sum_{j \in \mathcal{N}_{1}}\left(\mathbf{A}_{j}-\mathbf{A}_{1}+\mathbf{D}_{1 j}\right)+\sum_{j \in \mathcal{N}_{i}}\left(\mathbf{A}_{j}-\mathbf{A}_{i}\right. \\
& \left.+R\left(\phi_{i}\right) \mathbf{D}_{i j}\right)+R\left(\phi_{i}\right)\left(\sum_{j \in \mathcal{N}_{i}}\left(-\mathbf{D}_{i j}+R\left(-\hat{\phi}_{i}\right) \mathbf{D}_{i j}\right)\right) .
\end{aligned}
$$

It then follows that for all $i=2,3, \ldots, n, \dot{\mathbf{A}}_{i}-\dot{\mathbf{A}}_{1}=-\sum_{j \in \mathcal{N}_{1}}\left(\mathbf{A}_{j}-\right.$ $\left.\mathbf{A}_{1}+\mathbf{D}_{1 j}\right)+\sum_{j \in \mathcal{N}_{i}}\left(\mathbf{A}_{j}-\mathbf{A}_{i}+R\left(\phi_{i}-\hat{\phi}_{i}\right) \mathbf{D}_{i j}\right)$. Noting that we have shown from Theorem 7 that $\lim _{t \rightarrow \infty} R\left(\phi_{i}-\hat{\phi}_{i}(t)\right)=\mathbf{I}$, for all $i=2,3, \ldots, n$, it then follows from the input-to-state stability property (Khalil, 2002) that $\lim _{t \rightarrow \infty}\left(\mathbf{A}_{1}(t)-\mathbf{A}_{i}(t)\right)=\mathbf{D}_{1 i}$, for all $i=2,3, \ldots, n$. It is then trivial to show that $\lim _{t \rightarrow \infty} \mathbf{V}_{i}(t)=0$, for all $i \in \mathcal{V}$.

\section{Concluding remarks}

This article studied the formation control problem with mismatched compasses. Such a mismatch is a consequence of the fact that it is not physically realistic to claim that all agents have common error-free knowledge of where north is. We examined the consequences of the mismatched compasses on a standard formation control algorithm. The two agent case was first studied and we showed that the agents converge to a fixed, but distorted, formation exponentially fast. Unlike the matched compass case, the formation is not asymptotically stationary. The shape error between the actual final formation and the desired formation was explicitly established. We then studied the case of timevarying mismatched compasses and the estimation problem for the mismatch angle. Based on this analysis, the compensation algorithm was proposed such that the desired formation shape is achieved exponentially fast. The exponential property provides a measure of robustness to noise. The extensions to the $n$ agent case where $n \geq 3$ were followed. Simulations are provided to validate the theoretical results in the absence of noise. 
The results of this article raise a number of open issues, including considering other agent models, such as second order agents or unicycle and considering the effect of bias in distances simultaneously with mismatch in the direction of north. One could also seek to obtain an extension of the algorithms to cope with compensation where the initial angular misalignment was arbitrarily large.

\section{References}

Belabbas, M. A. Mou, S. Morse, A. S. \& Anderson, B. D. O. 2012. Robustness issues with undirected formations. In Proceedings of the 2012th IEEE conference on decision and control. (pp. 1445-1450) Maui, Hawaii, USA.

Cao, M., Morse, A. S., Yu, C., Anderson, B. D. O., \& Dasgupta, S. (2011). Maintaining directed, triangular formation of mobile autonomous agents. Communications in Information and Systems, 11(1), 1-16.

Cortes, J., Martinez, S., \& Bullo, F. (2006). Robust rendezvous for mobile autonomous agents via proximity graphs in arbitrary dimensions. IEEE Transactions on Automatic Control, 51(8), 1289-1298.

Fax, J. A., \& Murray, R. M. (2004). Information flow and cooperative control of vehicle formations. IEEE Transactions on Automatic Control, 49(9), 1465-1476.

Hatano, Y., \& Mesbahi, M. (2005). Agreement over random networks. IEEE Transations on Automatic Control, 50(11), 1867-1872.

He, W., Qian, F., Lam, J., Chen, G., Han, Q.-L., \& Kurths, J. (2015). Quasisynchronization of heterogeneous dynamic networks via distributed impulsive control: Error estimation, optimization and design. Automatica, 62, 249-262.

Jiang, B., Deghat, M., \& Anderson, B. D. O. 2013. Translational velocity consensus using distance-only measurements. In Proceedings of 2013th IEEE conference on decision and control. (pp. 2746-2751). Florence, Italy.

Khalil, H. K. (2002). Nonlinear systems. Prentice Hall.

Krick, L., Broucke, M. E., \& Francis, B. A. (2009). Stabilization of infinitesimally rigid formations of multi-robot networks. International Journal of Control, 82(3), 49-95.

Lin, Z., Broucke, M., \& Francis, B. (2004). Local control strategies for groups of mobile autonomous agents. IEEE Transactions on Automatic Control, 49(4), 622-629.

Meng, Z., Anderson, B. D. O., \& Hirche, S. (2015). Analysis of undirected formation shape control with directional mismatch. In 54th IEEE conference on decision and control (pp. 6773-6778). Japan: Osaka.

Moreau, L. (2005). Stability of multi-agent systems with time-dependent communication links. IEEE Transactions on Automatic Control, 50(2), 169-182.

Oh, K.-K., \& Ahn, H.-S. (2014). Formation control and network localization via orientation alignment. IEEE Transactions on Automatic Control, 59(2), 540-545.

Olfati-Saber, R., Fax, J. A., \& Murray, R. M. (2007). Consensus and cooperation in networked multi-agent systems. Proceedings of the IEEE, 95(1), 215-233.

Peng, K., \& Yang, Y. (2009). Leader-following consensus problem with a varyingvelocity leader and time-varying delays. Physica A, 388(2-3), 193-208.

Ren, W. (2007). Multi-vehicle consensus with a time-varying reference state. Systems E' Control Letters, 56(7), 474-483.

Shi, G., \& Hong, Y. (2009). Global target aggregation and state agreement of nonlinear multi-agent systems with switching topologies. Automatica, 45(5), $1165-1175$.

Sieber, D., Deroo, F., \& Hirche, S. 2013. Formation-based approach for multirobot cooperative manipulation based on optimal control design. In IEEE/RSJ international conference on intelligent robots and systems (IROS). (pp. 5227-5233) Tokyo, Japan.

Sun, Z., Mou, S., Anderson, B. D. O., \& Morse, A.S. 2013. Non robustness of gradient control for 3-d undirected formations with distance mismatch. In proceedings of 2013th Australian control conference. (pp. 369-374) Perth, Australia.

Yang, T., Roy, S., Wan, Y., \& Saberi, A. (2011). Constructing consensus controllers for networks with identical general linear agents. International Journal of Robust and Nonlinear Control, 21(11), 1237-1256.

Zavlanos, M. M., \& Pappas, G. J. (2008). Distributed connectivity control of mobile networks. IEEE Transactions on Robotics, 24(6), 1416-1428.

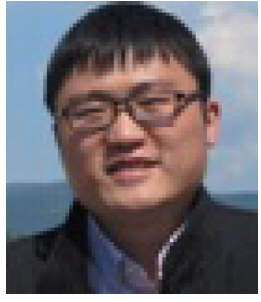

Ziyang Meng received his Bachelor degree with honors from Huazhong University of Science \& Technology, Wuhan, China, in 2006, and Ph.D. degree from Tsinghua University, Beijing, China, in 2010. He was an exchange Ph.D. student at Utah State University, Logan, USA from Sept. 2008 to Sept. 2009. From 2010 to 2015, he held postdoc, researcher, and Humboldt research fellow positions at, respectively, Shanghai Jiao Tong University, Shanghai, China, KTH Royal Institute of Technology, Stockholm, Sweden, and Technical University of Munich, Munich, Germany. He joined Department of Precision Instrument, Tsinghua University, China as an associate professor since Sept. 2015. He was selected in the Guan Zhaozhi Best Paper Award Finalist from Chinese Control Conference in 2014 and selected to the national "1000-Youth Talent Program" of China in 2015. His research interests include multiagent systems, small satellite systems, distributed control and optimization, nonlinear systems and information fusion techniques.

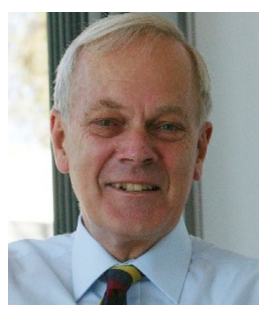

Brian D.0. Anderson was born in Sydney, Australia, and educated at Sydney University in mathematics and electrical engineering, with Ph.D. in electrical engineering from Stanford University in 1966. Following graduation, he joined the faculty at Stanford University and worked as Vidar Corporation of Mountain View, California, as a staff consultant. He then returned to Australia to become a department chair in electrical engineering at the University of Newcastle. From there, he moved to the Australian National University in 1982, as the first engineering professor at that university. He is now a Distinguished Professor at the Australian National University and Distinguished Researcher in National ICT Australia. During his period in academia, he spent significant time working for the Australian Government, with this service including membership of the Prime Minister's Science Council under the chairmanship of three prime ministers. He also served on advisory boards or boards of various companies, including the board of the world's major supplier of cochlear implants, Cochlear Corporation, where he was a director for ten years. His awards include the IFAC Quazza Medal in 1999, IEEE Control Systems Award of 1997, the 2001 IEEE James H Mulligan, Jr Education Medal, and the Bode Prize of the IEEE Control System Society in 1992, as well as IEEE and other best paper prizes, including one from Automatica. He is a Fellow of the Australian Academy of Science, the Australian Academy of Technological Sciences and Engineering, the Royal Society (London), and a foreign member of the US National Academy of Engineering. He holds honorary doctorates from a number of universities, including Université Catholique de Louvain, Belgium, and ETH, Zürich. He served as IFAC President from 1990 to 1993, having had earlier periods in various IFAC roles, including editor of Automatica. He was also President of the Australian Academy of Science from 1998 to 2002. His current research interests are in distributed control, sensor networks and econometric modeling.

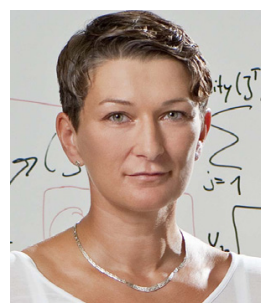

Sandra Hirche received the Diplom-Ingenieur degree in aeronautical engineering from Technical University Berlin, Germany, in 2002 and the Doktor-Ingenieur degree in electrical engineering from Technical University Munich, Germany, in 2005. From 2005 to 2007 she was awarded a Postdoc scholarship from the Japanese Society for the Promotion of Science at the Fujita Laboratory, Tokyo Institute of Technology, Tokyo, Japan. From 2008 to 2012 she has been an associate professor at Technical University Munich. Since 2013 she is TUM Liesel Beckmann Distinguished Professor and has the Chair of Information-oriented Control in the Department of Electrical and Computer Engineering at Technical University Munich. Her main research interests include cooperative, distributed and networked control with applications in robotics and smart infrastructure systems. 